\title{
On-line impact load identification
}

\author{
Krzysztof Sekuła*, Cezary Graczykowski and Jan Holnicki-Szulc \\ Institute of Fundamental Technological Research, Warsaw, Poland
}

Received 1 December 2011

Revised 29 May 2012

\begin{abstract}
The so-called Adaptive Impact Absorption (AIA) is a research area of safety engineering devoted to problems of shock absorption in various unpredictable scenarios of collisions. It makes use of smart technologies (systems equipped with sensors, controllable dissipaters and specialised tools for signal processing). Examples of engineering applications for AIA systems are protective road barriers, automotive bumpers or adaptive landing gears. One of the most challenging problems for AIA systems is on-line identification of impact loads, which is crucial for introducing the optimum real-time strategy of adaptive impact absorption. This paper presents the concept of an impactometer and develops the methodology able to perform real-time impact load identification. Considered dynamic excitation is generated by a mass $M_{1}$ impacting with initial velocity $V_{0}$. An analytical formulation of the problem, supported with numerical simulations and experimental verifications is presented. Two identification algorithms based on measured response of the impacted structure are proposed and discussed. Finally, a concept of the AIA device utilizing the idea of impactometer is briefly presented.
\end{abstract}

Keywords: Impact load identification, Adaptive Impact Absorption, adaptive structures, real-time systems, impact tests

\section{Introduction}

The objective of this paper is strongly related to the concept of Adaptive Impact Absorption (AIA) [1-3]. An AIA system is a structure equipped with control devices that modify its local structural properties (e.g. local stiffness) in real-time in order to adapt the structure to the actual dynamic loading. The initial challenge is to invent the technologies applicable to the above-mentioned control devices. One option deals with the concept of structural fuses with elasto-plastic type of overall performance and the controllable yield stress level, where the control mechanism can be based on various types of actuators, e.g. electromagnetic, piezoelectric, magnetostrictive or magneto Shape Memory Alloys (SMA) [4,5]. Shock absorbers based on magneto-rheological fluids or piezoelectric valves can be successfully utilised for AIA in case of repetitive operational impacts, for example in adaptive landing gears [6]. Another type of AIA systems are Adaptive Inflatable Structures (AIS) [7] composed of chambers filled with compressed gas and equipped with high performance valves which control the release of pressure during impact. Finally, Micro Pyro-technique Systems (MPS) can be used for detaching (in real-time) selected structural joints in order to improve the structural response in emergency situations (e.g. in crash of vehicles) [8].

Examples of engineering applications for AIA systems are protective road barriers $[9,10]$, automotive: longitudinal members [11], bumpers [12], head-rest [13], so-called multi-folding protective systems [12], adaptive landing gears [2,14], pneumatic offshore structures [7] and adaptive airbags for emergency landings [15]. The general overview of the AIA concepts developed in Institute of Fundamental Technological Research can be found in [16, 17]. In all mentioned applications, the development of the optimal adaptation strategy requires information about the characteristics of the impact loading and the value of the impact energy which has to be dissipated. Therefore, the initial step of the adaptive impact absorption should be identification of the impact loading.

\footnotetext{
*Corresponding author: Krzysztof Sekuła, Institute of Fundamental Technological Research, Warsaw, Poland. E-mail: ksekula@ippt.gov.pl.
} 

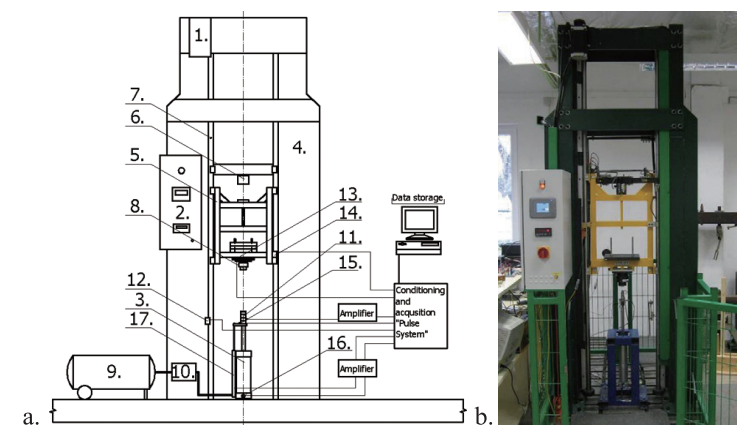

Fig. 1. Experimental free-fall drop test stand: a. schema of the set-up; b. view of the test stand.
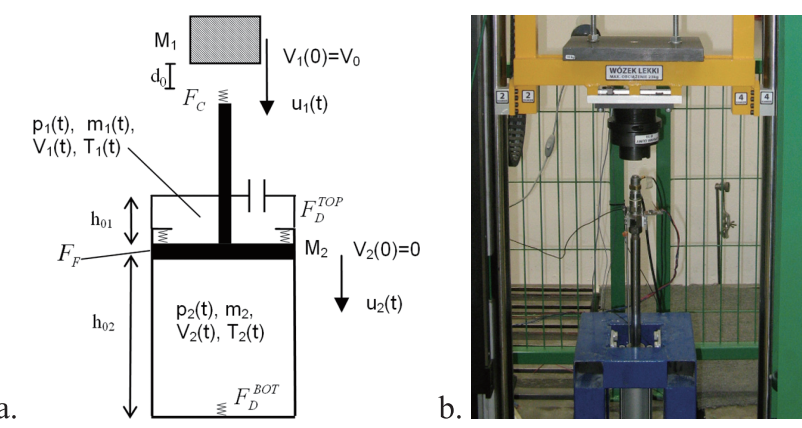

Fig. 2. Considered mechanical system: a. numerical model; b. experimental set-up.

This paper is focused on the problem of real-time identification of impact load (the impacting mass and its initial velocity). The identification is performed by means of a specially invented device, the so-called impactometer (patent pending) which is equipped with a force sensor (and/or accelerometer) and a tuneable gas-spring. It is assumed that the impactometer is located on the structure protected by the AIA system and that is exposed to impacts of objects of unknown masses and velocities.

Many techniques were developed in order to identify parameters of impact and dynamic excitations. This methodology usually belongs to the class of inverse problems. Similar to Structural Health Monitoring (SHM) [32], impact identification problem can be defined as finding system inputs, based on given responses, boundary conditions and system model [33]. Generally identification techniques can be divided into three main categories: deterministic, stochastic and based on artificial intelligence [34,35]. Impact identification techniques are needed for different technical application e.g. aircraft landing gears [2,14], composites structures [35,36] etc. In [18], a brief review of methods used in the case of indirect impact force identification is presented. The article considers a variety of approaches for identification of the time history of an impact force, its direction and location. The authors of [19] give a detailed overview of the on-line load identification techniques. Moreover, the paper [20] points out that the load identification problem is fundamentally an experimental one and requires good quality data for its successful solution.

Taking into account the objective of this paper, the crucial issue is the operation time criterion (i.e. the time required to identify parameters of the impact load) which has to satisfy the deadline condition. The dead-line condition is to identify the impact parameters at the very beginning of the process i.e. before the time instant when the acceleration of the colliding object achieves its optimal value (the constant value that enables to use the full stroke of the controllable absorber). Contrary to the off-line identification techniques, cf. e.g. [21], which usually make use of a numerical model of structure and require long computational time, the identification techniques considered in that paper have to be performed in a few milliseconds to allow the AIA control procedure to be triggered in real-time. The developed identification algorithms utilize sensors which are not directly fixed to the impacting object. It is justified by the practical aspects and potential application of the impactometer for unidentified impacting objects parameter's estimation.

The layout of the paper is as follows. In the second section, the laboratory set-up for drop tests is demonstrated. In the third section, numerical model for simulation of the dynamic response of the impactometer device is developed. Results of the experimental tests and numerical simulations for the series of various impact scenarios are presented in Section 4. In the following fifth section, an analytical algorithm for impact load identification is formulated and verified numerically as well as experimentally. Finally, in Section 6 another algorithm, based on response map approach is presented and verified. The plans for implementation of the impactometer concept are described in Section 7 while the concluding remarks are drown in the last section.

\section{Drop testing stand}

Impact tests have been performed using a free-fall drop test stand (Fig. 1). The set-up allows to generate initial impact energy up to $1.5 \mathrm{~kJ}$ by a mass of $100 \mathrm{~kg}$ dropped from the height of $1.5 \mathrm{~m}$. A pneumatic absorber equipped 
with sensors has been used as a prototype of the impactometer. Adjustment of initial pressure inside the cylinder has been found to be an effective method of modification of its properties. Moreover, a controllable exhaust valve installed into the pneumatic cylinder allows the impactometer to act as a semi-actively controlled impact energy absorber whose characteristics can be modified relatively simply and quickly.

The test-stand is equipped with electric motor (1) for lifting the mass and a control switch box (2) used to program a set of drop tests. The main parts of the set-up are the pneumatic cylinder (3) mounted in the vertical position (diameter $63 \mathrm{~mm}$, maximum stroke $250 \mathrm{~mm}$ ), the frame (4) and the carriage (5). The lifting mechanism includes an electromagnet (6) used for releasing the impacting mass fixed to the carriage (5), which is guided by the rail system (7) embedded in the frame. The mass is impacting onto the pneumatic cylinder via a rubber bumper (8). The compressor (9) enables to modify the initial pressure inside the pneumatic cylinder (3) via the regulator (10). The measurement system includes all necessary conditioning systems and amplifiers. The measured data, which are recorded at $50 \mathrm{kHz}$ by a Pulse (Brüel \& Kjær) acquisition system, include signals from:

- the piezoelectric sensor (11) fixed to the piston rod of the pneumatic cylinder, in order to measure the impact force-time history,

- the optical switch (12), which acts as a trigger and allows to determine the vertical velocity of the impacting mass just before the impact,

- the accelerometer attached to the carriage to determine deceleration of the falling mass (13),

- the magnetic linear sensor (14) to determine displacement of the falling mass,

- the accelerometer (15) attached to the piston rod of the pneumatic cylinder,

- the "fast" pressure sensor (16) mounted in the cylinder,

- the LVDT sensor (17) to determine displacement of the piston.

\section{Numerical model of the system}

The pneumatic absorber described above was modelled numerically in order to simulate its response to various impact scenarios, i.e. impacts of a rigid object of various masses and initial velocities. Another purpose of numerical simulations was to estimate the influence of selected parameters of the pneumatic cylinder on the dynamic response of the system and further to choose the optimal parameters of the impactometer.

The proposed basic model of a pneumatic absorber subjected to impact loading is based on the assumption that pressure, density and temperature of the gas in each chamber are uniform in every instant of time. Moreover, due to the fact that considered impact process is relatively short it is assumed that the heat transfer through the cylinder walls does not occur and so the process can be considered as adiabatic. The numerical model is schematically presented in Fig. 2(a), while the corresponding mechanical system is shown in Fig. 2(b).

The considered system consists of two rigid objects, the falling mass and the piston, which are represented by two mechanical degrees of freedom. Corresponding equations of motion take the form:

$$
\begin{aligned}
& M_{1} \frac{d^{2} u_{1}}{d t^{2}}-M_{1} g+F_{C}=0 \\
& M_{2} \frac{d^{2} u_{2}}{d t^{2}}-M_{2} g-F_{C}+F_{P}+F_{F}-F_{D}^{T O P}+F_{D}^{B O T}=0
\end{aligned}
$$

where: $M_{1}, M_{2}$ are the masses of the falling object and the piston; $u_{1}, u_{2}$ denote their displacements and $g$ is the gravitational acceleration. The equation of piston motion Eq. (2) contains terms denoting the following forces: pneumatic $F_{P}$, the friction $F_{F}$ and two delimiting forces $F_{D}^{T O P}, F_{D}^{B O T}$. Equations (1) and (2) are coupled by the contact force $F_{C}$ which acts between the falling mass and the piston rod. The contact force $F_{C}$ depends on the material properties of the colliding objects and moreover, it is influenced by the geometry and shape of contact surfaces. Simplified models of the unidirectional contact proposed in the literature [22] usually include some combination of springs with stiffness $k$ and dampers with viscous damping $c$. Let us recall here the following classical models described by the Eq. (3a,b) where the initial distance between bodies is denoted by $d_{0}$, while $u_{1}, u_{2}, \dot{u}_{1}, \dot{u}_{2}$ denote the displacements and velocities of falling object and the piston: 
- linear visco-elastic Kelvin-Voigt model

$$
F_{C}=k\left(u_{1}-u_{2}-d_{0}\right)+c\left(\dot{u}_{1}-\dot{u}_{2}\right)
$$

- model with linear spring and nonlinear dashpot

$$
F_{C}=k\left(u_{1}-u_{2}-d_{0}\right)+c\left(u_{1}-u_{2}-d_{0}\right)\left(\dot{u}_{1}-\dot{u}_{2}\right)
$$

A thorough analysis of contact force models will be presented in Section 5.2 that concerns the "peak-to-peak approach", since the assumed contact model will be important for that identification procedure.

The pneumatic force $F_{P}$ is defined as the difference of the pneumatic forces that act on both sides of the piston. These forces results from pressures $p_{1}$ and $p_{2}$ acting on upper and lower piston area $\left(A_{1}\right.$ and $\left.A_{2}\right)$, as well as from ambient pressure acting on the piston rod:

$$
F_{P}=p_{2} A_{2}-p_{1} A_{1}-p_{A}\left(A_{2}-A_{1}\right)
$$

The friction force $F_{F}$ models the friction between the piston and cylinder walls. Two models were considered: the Coulomb friction, which depends only on the direction of piston movement, and a velocity-dependent friction described by a friction coefficient $c_{F}$ :

$$
\left\{F_{F}=-F \quad \text { if } \quad \frac{d u_{2}}{d t}<0 \quad \text { and } \quad F_{F}=F \quad \text { if } \quad \frac{d u_{2}}{d t}>0\right\} \quad \text { or } \quad\left\{F_{F}=c_{F} \frac{d u_{2}}{d t}\right\}
$$

In order to confine the piston movement to the range determined by the cylinder geometry, the top delimiting force $F_{D}^{T O P}$ and the bottom delimiting force $F_{D}^{B O T}$ were used. Both forces are defined in the simplified form as linear springs with the stiffness $k_{D}$. The bottom delimiting force $F_{D}^{B O T}$ is generated when the piston reaches position $u_{2}^{\text {crit }}$.

$$
\begin{aligned}
& F_{D}^{T O P}=-k_{D} u_{2} \quad \text { if } u_{2}<0 \text { and } F_{D}^{T O P}=0 \quad \text { if } u_{2} \geqslant 0 \\
& F_{D}^{B O T}=k_{D}\left(u_{2}-u_{2}^{\text {crit }}\right) \text { if } u_{2}>u_{2}^{\text {crit }} \text { and } F_{D}^{B O T}=0 \quad \text { if } u_{2} \leqslant u_{2}^{\text {crit }}
\end{aligned}
$$

The following part of the numerical model is related directly to thermodynamics of the system. The basic equation describing gas in both chambers is the ideal gas law:

$$
\begin{array}{lll}
p_{1} V_{1}=m_{1} R T_{1} & \text { where } & V_{1}=A_{1}\left(h_{01}+u_{2}\right) \\
p_{2} V_{2}=m_{2} R T_{2} & \text { where } & V_{2}=A_{2}\left(h_{02}-u_{2}\right)
\end{array}
$$

where: $V_{1}, V_{2}, T_{1}, T_{2}$ denote the volumes of the chambers and their temperatures, $R$ is the gas constant, $m_{1}, m_{2}$ are air masses while $h_{01}, h_{02}$ are the initial lengths of the chambers. The flow of the gas between the upper chamber and the environment can be described by a simple formula which relates mass flow rate of gas into the upper chamber with the pressure difference between the chamber and environment:

$$
\Delta p=p_{1}-p_{A}=-C_{V}(t) \dot{m}_{1}-C_{H}(t) \dot{m}_{1}\left|\dot{m}_{1}\right|
$$

where: $C_{V}, C_{H}$ are the flow resistance coefficients. Other, more sophisticated analytical models of the gas flow can be found in the classical literature [23,24]. However, let us note that precise modelling of flow of the gas to the upper chamber of the cylinder is beyond the scope of this paper. The inflow of the gas could be totally blocked or totally opened with no significant influence on further considerations and the conclusions concerning impact identification.

Moreover, balance of internal energy of gas enclosed in each chamber has to be considered. General form of equation of internal energy balance for an open chamber with adiabatic walls and variable volume involves enthalpy of gas added/removed from the chamber, change of gas internal energy and work done by gas [25]:

$$
d m_{\text {in }} \bar{H}_{\text {in }}-d m_{\text {out }} \bar{H}_{\text {out }}=d(m \bar{U})+d W
$$

Specific gas enthalpy $\bar{H}_{i n} / \bar{H}_{\text {out }}$, specific gas energy $\bar{U}$ and work done by gas $W$ are defined as follows:

$$
\bar{H}_{\text {in }}=c_{p} T_{\text {in }} ; \quad \bar{H}_{\text {out }}=c_{p} T ; \quad \bar{U}=c_{V} T ; \quad d W=p d V
$$

where: $c_{p}$ and $c_{V}$ denote specific heat at constant pressure and constant volume, and $T_{i n}$ denotes temperature of the gas which enters the chamber. For the upper chamber Eq. (9) has to be considered in a full form. By contrast, for the 

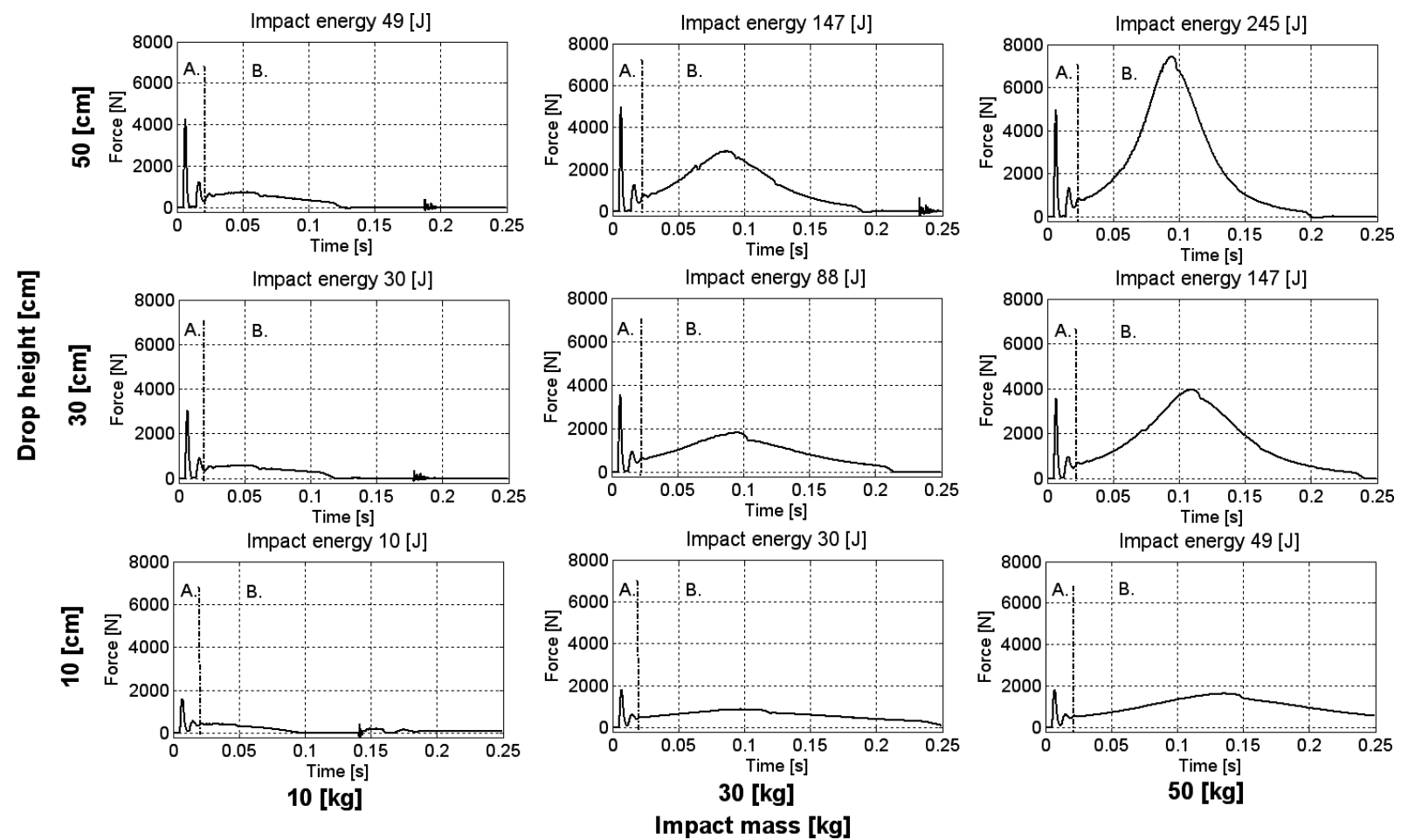

Fig. 3. The effect of impact mass and velocity on the contact force time-history for initial pressure $100 \mathrm{kPa}$.

lower chamber which remains closed the term denoting enthalpy vanishes and differential Eq. (9) simplifies to the algebraic one.

The initial conditions for the system are defined by the initial position and velocity of the falling object and the piston, and the initial parameters of the gas inside the cylinder:

$$
\begin{aligned}
& u_{1}(0)=u_{1}^{0}, \quad \frac{d u_{1}}{d t}(0)=V_{1}^{0}, \quad u_{2}(0)=u_{2}^{0}, \quad \frac{d u_{2}}{d t}(0)=0, \\
& p_{1}(0)=p_{A}, \quad T_{1}(0)=T_{1}^{0}, \quad p_{2}(0)=p_{2}^{0}, \quad T_{2}(0)=T_{2}^{0}
\end{aligned}
$$

Equations (1-11) fully define the problem of a double chamber pneumatic cylinder subjected to an impact loading. The variables for which the equations are solved (chosen among $u_{1}, u_{2}, m_{1}, m_{2}, p_{1}, T_{1}, p_{2}, T_{2}$ ) and the corresponding initial conditions can be chosen arbitrarily. The final system of differential equations was implemented in mathematical software MAPLE and solved numerically by the fourth-order Runge-Kutta method.

\section{Impact scenarios}

This section presents examination of the impact process for the considered mechanical system. The objective is to analyze the structural response under impact excitation with regard to collision parameters as well as impact absorber properties. The considerations are performed on the basis of the experimental and numerical results.

\subsection{Experimental tests of impact scenarios}

The considered impact types are limited to the collinear central collision between the rigid bodies (i.e. falling mass and the piston rod of the pneumatic absorber). A variety of impact scenarios of this kind have been tested. The impact scenarios were defined by three parameters: the impacting mass value, the velocity of the colliding object and the initial pressure inside the pneumatic absorber. The range of the impacting mass was $10-55 \mathrm{~kg}$, while 

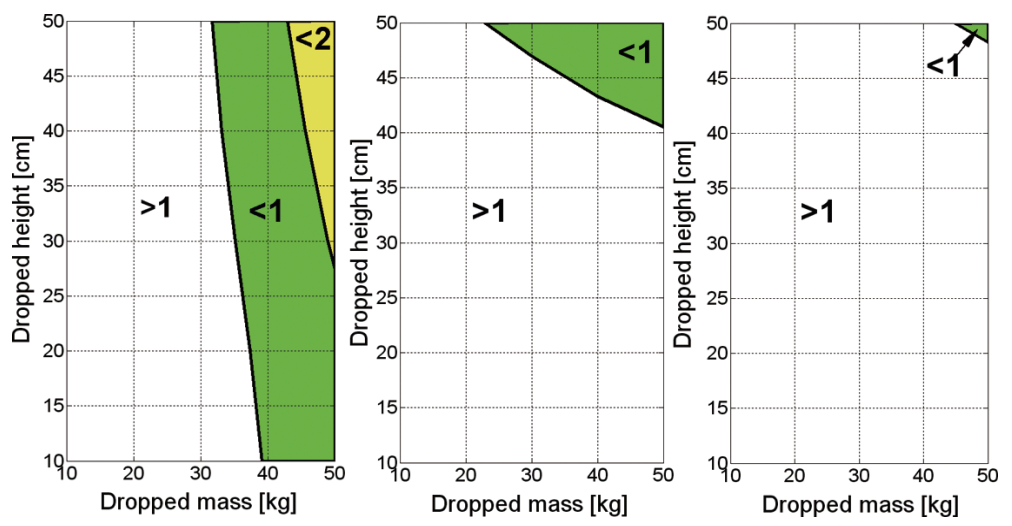

Fig. 4. Ratio of maximal forces in two stages of the process in the mass velocity area for different initial pressures: $20 \mathrm{kPa}$ (left), $100 \mathrm{kPa}$ (middle), $200 \mathrm{kPa}$ (right).

the relative impact velocity was adjusted by the drop height, which was confined to the range of $0.05-0.5 \mathrm{~m}$. The pressure parameter was modified in the range of $0-400 \mathrm{kPa}$ where 0 is understood as the atmospheric pressure.

The chosen cases of collision scenarios are presented in Fig. 3. The graphs illustrate the experimental measurements of the contact force acting between the impacting mass and the piston rod.

Characteristic change of contact force observed in the experiment allows to divide the impact process into two separate stages (i.e. $A, B$ ) which were marked in Fig. 3:

- the first one i.e. stage $(A)$ when the piston rod rebounds from the falling mass and large oscillations of the contact force occur,

- the second one i.e. stage $(B)$ during which the falling mass is moving downwards together with the piston which results in a smooth change of the contact force.

The total duration of the stage $(A)$ was approx. 20-40 ms (mainly depending on the initial pressure), while the duration of an average force peak was approx. $5 \mathrm{~ms}$. The phenomenon of the initial piston rebounds in stage $(A)$ is the consequence of a relatively small value of force acting on the bottom part of the piston at the beginning of the process. Each of the considered impacts can be characterized by the number of rebounds between the falling mass and the piston rod observed in the stage $(A)$ of the process. For the employed range of impact conditions (massvelocity-initial pressure) it was found that the number of the rebounds was between $0-2$. It might be concluded that the most important factor for occurrence of the rebounds was the initial pressure. In general, the maximum number of rebounds was observed in the case of the smallest initial pressure. Generally, the stage $(A)$ of the process reveals strong sensitivity to impact velocity but appears to be only slightly dependent on the mass of the hitting object. The first peak of the contact force increases together with the impact velocity which is the result of the explicit dependency of the contact force on velocity of the impacting object (cf. Eq. (3).)

In further part of the process (i.e. stage B), the falling mass is moving downwards together with the piston. The stage $B$ is significantly sensitive to both impact velocity and mass of the hitting object. Some of the collisions presented in Fig. 3 are characterized by the same impact energy, for example $(10 \mathrm{~kg}-50 \mathrm{~cm}$ and $50 \mathrm{~kg}-10 \mathrm{~cm}) . \mathrm{In}$ these cases, very different structural responses were observed, as defined by the force time-history in both impact stages. The impact of the same energy caused by an object with a larger mass results in a higher value of the contact force in the second stage of the impact. It is mainly caused by the impacting object vertical movement and the effect of change of its potential energy via the absorber compression.

The objective of the considered problem of impact identification is to utilize the measurements performed during stage $(A)$ to obtain the desired impact parameters. This would allow the stage $(B)$ of the impact process to be controlled in order to reduce the impact force and the acceleration of the colliding objects (the so called Adaptive Impact Absorption, cf. [1]). The proposed methodology requires that the maximal value of the contact force in stage $(A)$ is lower than the maximal value of contact force in stage $(B)$. The above condition was examined for presented experimental data and it results in contour lines shown in Fig. 4. For each value of the initial pressure, the plane defined by the impact parameters (impacting mass and drop height) can be divided into several areas defined by 


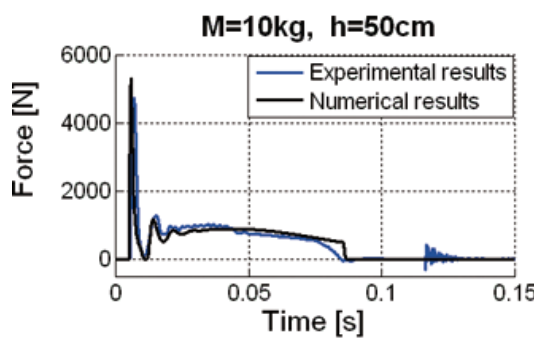

$M=10 \mathrm{~kg}, \mathrm{~h}=30 \mathrm{~cm}$

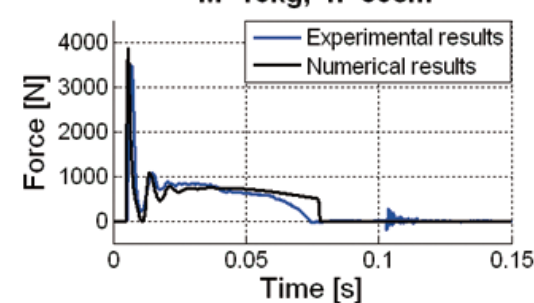

$M=10 \mathrm{~kg}, h=10 \mathrm{~cm}$

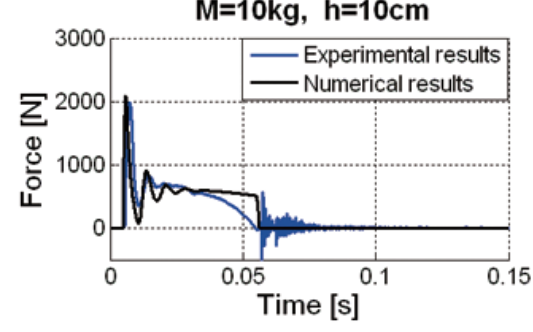

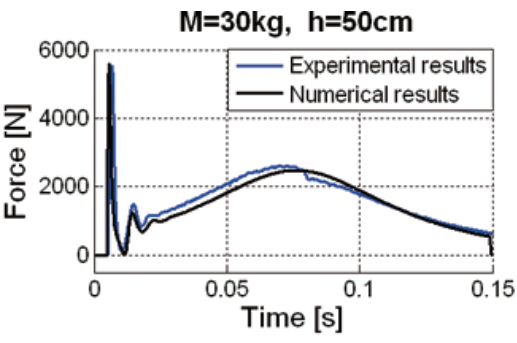

$M=30 \mathrm{~kg}, \mathrm{~h}=30 \mathrm{~cm}$

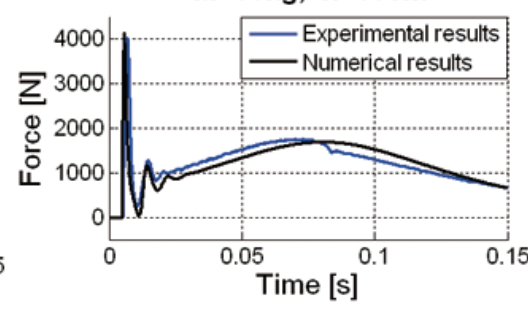

$M=30 \mathrm{~kg}, h=10 \mathrm{~cm}$

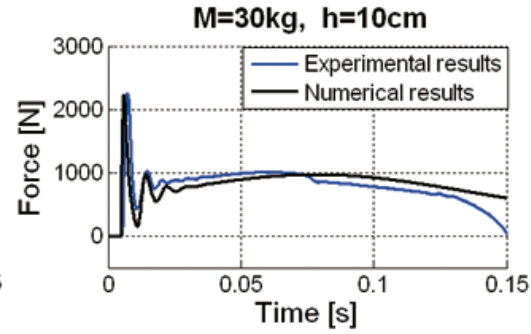

$M=50 \mathrm{~kg}, \mathrm{~h}=50 \mathrm{~cm}$

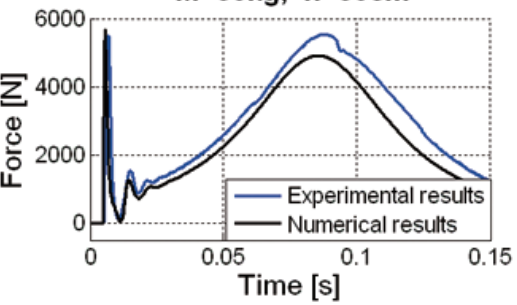

$M=50 \mathrm{~kg}, h=30 \mathrm{~cm}$

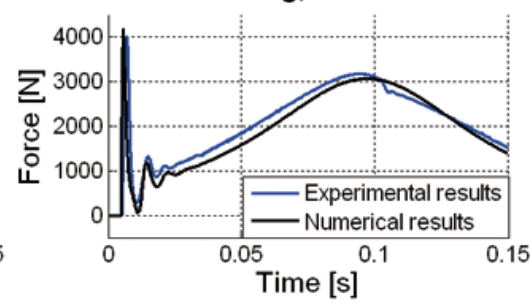

$M=50 \mathrm{~kg}, \mathrm{~h}=10 \mathrm{~cm}$

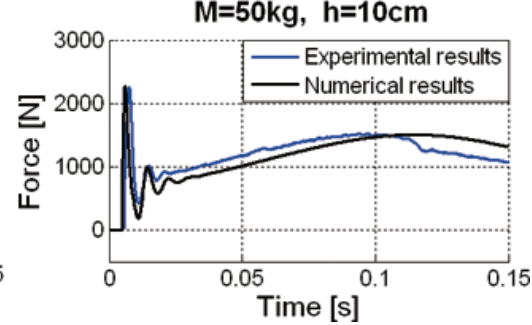

Fig. 5. Comparison of experimental and numerical results of contact force in time domain (initial pressure equal $200 \mathrm{kPa}$ ).

ratio of the maximal force in first and second stages of the process. It can be concluded that for the experimentally tested impact conditions low initial pressure is more profitable for the proposed methodology of combined impact identification and absorption.

\subsection{Numerical simulation of impact scenarios}

Comparison of the numerical results obtained on the basis of the model described in Section 3 and the experimental measurements, is presented in Fig. 5. Although the results are in good qualitative agreement, the exact quantitative compatibility for the wide range of impact scenarios is difficult to obtain. The main reasons are complex and difficult to estimate mechanical properties of the rubber bumper used in the experimental set up (hyperelastic material properties including Mullins and Payne effects [26,27]). In general, the complexity of the model should depend on the objectives of the analysis to be performed. Here, that the model was used mainly to examine the qualitative influence of selected parameters. The attention for the numerical model validation was especially focused on stage $A$ of the impact process when the identification process is going to be performed.

Another purpose of the numerical model was an examination of the dynamic responses of the pneumatic system subjected to the excitation with higher impact energies. The range of the considered impact scenarios was extended (in comparison to the experimental section) to the mass range 10-190 kg and height 10-190 cm. The results of the analysis for selected impact scenarios are presented in Fig. 6.

The most important conclusion from the conducted numerical analysis is that an increase of both the impacting object mass and the drop height (i.e. impact velocity) causes increase of the ratio of the peak contact force in stage $B$ to peak contact force in stage A. This ratio is presented in Fig. 7 in the form of a contour plot in terms of impacting mass and drop height. The good quantitative agreement with the experimental plot (see Fig. 4) was obtained. For high impact energies the ratio is even greater than five which indicates that an application of the proposed system of impact identification is justified. 

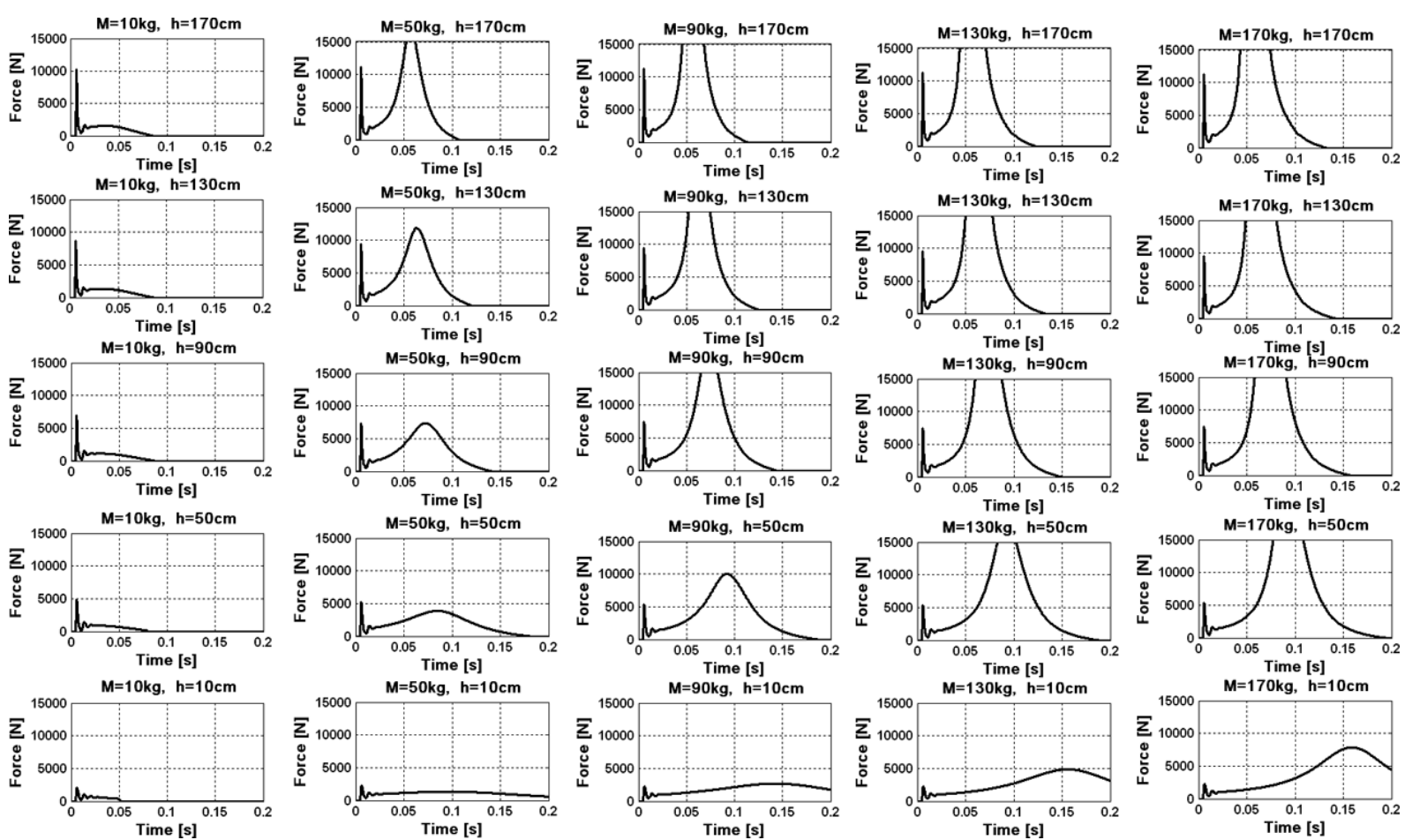

Fig. 6. Effect of impact mass and velocity on the contact force time-history (initial pressure $100 \mathrm{kPa}$ ).
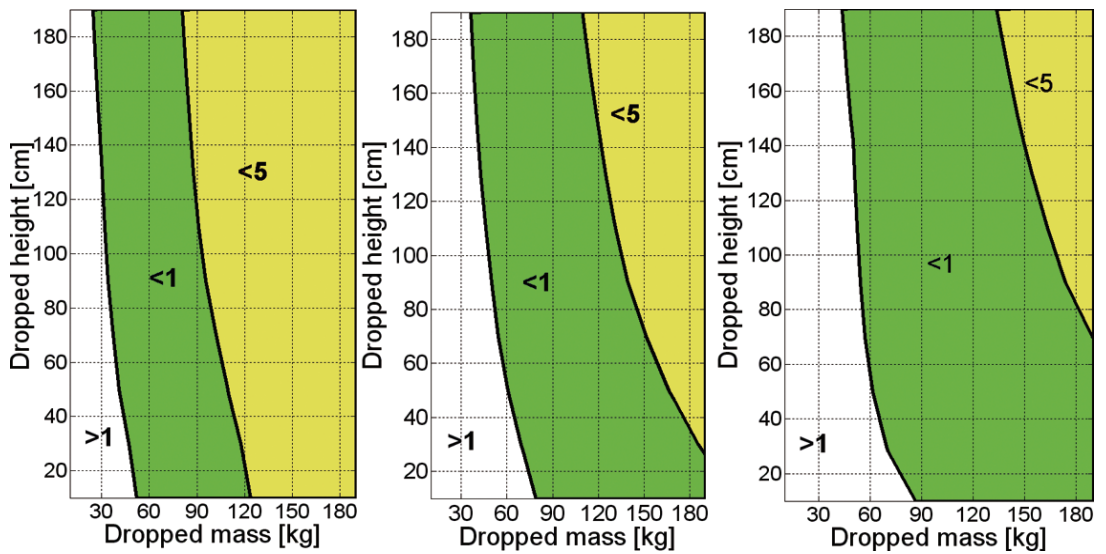

Fig. 7. Ratio of maximal forces in two stages of the process in dependence on the impact mass and velocity for different initial pressures: $20 \mathrm{kPa}$ (left), $200 \mathrm{kPa}$ (middle), $400 \mathrm{kPa}$ (right).

\section{Impact identification based on "peak-to-peak" approach}

The methodology presented in this section is based on well-known mechanical principles. It utilizes measurements from two sensors: force sensor and accelerometer. Alternatively, the identification is feasible with measurements from one sensor, however a well-tuned model of the absorption system is required.

\subsection{The idea}

As described in a previous section, the impact process begins with several rebounds of the piston and the falling mass. The rebounds are separated by short time periods during which both objects remain in contact with each other. 

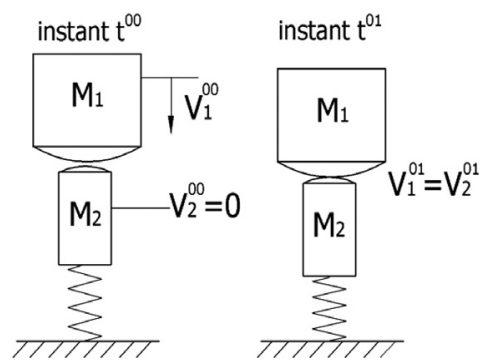

instant $\mathrm{t}^{02}$

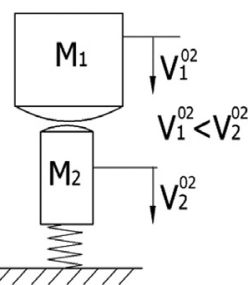

Fig. 8. Different stages of impact: (left) beginning of the compression stage, (middle) time instant when both velocities are equal, (right) end of the restitution stage.

During these periods certain instants of time must occur, when the velocities of both colliding objects are equal. This phenomenon is schematically shown in Fig. 8, where a simplified two degrees of freedom system is presented. The instants of time shown in Fig. 8 are also marked in Fig. 9(a).

The main idea of the proposed method of impact identification is based on computing the integral of the equation of motion of the impacting mass over time in the range defined by the time instants when the relative velocity of colliding objects vanishes. Let us denote these characteristic time instants by $t^{01}$ and $t^{11}$ (see Fig. 9). In the equations presented in this section the upper indices represent the instant of time while the lower ones correspond to the colliding objects. Integration of the equation of motion of the falling mass yields:

$$
M_{1} \int_{t 01}^{t^{11}} \ddot{u}_{1} d t-\int_{t 01}^{t^{11}} M_{1} g d t+\int_{t 01}^{t^{11}} F_{C}(t) d t=0
$$

and therefore

$$
M_{1}=-\frac{\int_{t^{01}}^{t^{11}} F_{C} d t}{\int_{t 01}^{11}\left(\ddot{u}_{1}-g\right) d t}=-\frac{\int_{t 01}^{t^{11}} F_{C} d t}{\left(V_{1}^{11}-V_{1}^{01}\right)-g \Delta t} .
$$

Taking the advantage of the fact that in the considered time instants the velocities of both objects are equal, the velocities and accelerations of the falling mass can be replaced by the velocities and accelerations of the piston:

$$
M_{1}=-\frac{\int_{t 01}^{t^{11}} F_{C}(t) d t}{\left(V_{2}^{11}-V_{2}^{01}\right)-g \Delta t}=-\frac{\int_{t 01}^{t^{11}} F_{C}(t) d t}{\int_{t_{01}^{11}}\left(\ddot{u}_{2}-g\right) d t} .
$$

The velocity of the impacting object at time instants $t^{01}$ and $t^{11}$ is simply determined based on the condition that both velocities are equal. Hence the following equations can be introduced:

$$
V_{1}^{01}=V_{2}^{01}=\int_{t 00}^{t^{01}} \ddot{u}_{2} d t, \quad V_{1}^{11}=V_{2}^{11}=\int_{t 00}^{t^{11}} \ddot{u}_{2} d t .
$$



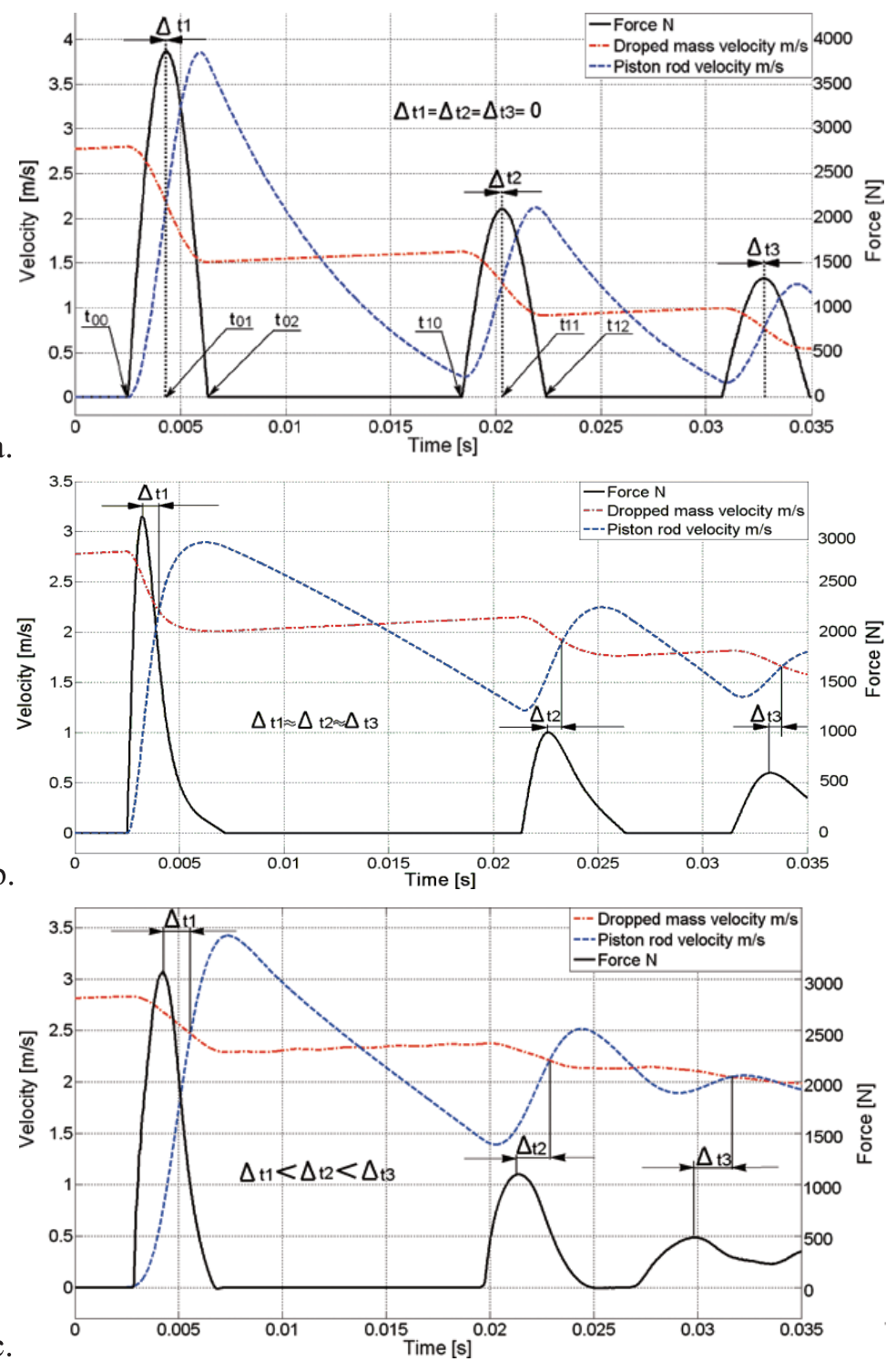

Fig. 9. Contact force and colliding object velocities in time domain for different models of the contact material: a. elastic contact force; b. contact force with nonlinear damping component; c. experimental results.

\subsection{Determination of time instants when colliding objects relative velocity vanishes}

In order to apply the methodology presented in the previous section, it is important to determine the instants $t^{01}$ and $t^{11}$. In Ref. [22] it is asserted that the relative velocity of contact points vanishes when the compression phase terminates and the restitution begins. In the classical impact theory (see ref. [28]) it is assumed that it occurs when the maximum contact force of colliding objects is observed. However, the following conditions have to be fulfilled:

- the contact force must increase in terms of displacement,

- the contact force must be mostly a function of the elastic deformation of colliding bodies and should be independent or negligibly dependent on other factors like velocity.

In general, the method of determination of time instants at which the velocities of both objects are equal depends on the properties of the contact element located between the falling mass and the piston. The three following cases will be considered separately: 
- elastic model of the contact force,

- model with viscous damping,

- real rubber applied in the experiment.

\subsubsection{Elastic model of contact force}

The assumption of contact force being purely elastic is often applied in the classical impact theory [22]. In such a case, the contact force arising between the falling object and the piston is a function of the distance between the centers of both bodies, i.e.:

$$
F_{C}=k x^{n}, \quad x=u_{1}-u_{2}-d_{0} \quad \text { if } \quad x>0,
$$

where $d_{0}$ indicates the initial distance between both objects while $u_{1}, u_{2}$ represent their displacements. The condition of the extremum of the contact force is defined by the equation:

$$
\frac{d F_{C}}{d t}=k n\left(u_{1}-u_{2}-d_{0}\right)^{n-1}\left(\dot{u}_{1}-\dot{u}_{2}\right)
$$

The above formula indicates that the velocities of both objects are equal exactly at the instant of time when the contact force reaches its extremum (i.e. maximum or minimum), see Fig. 9(a). Therefore, in case of elastic definition of the contact force the characteristic time instant, which have to be applied in the proposed method, can be determined very easily. An advantage of this method is that, except for the fact that the contact force is elastic, neither the exact knowledge of the constitutive relation nor the knowledge of exact value of the stiffness coefficient is required.

\subsubsection{Contact force with the viscous damping component}

In more general case the contact force is a linear combination of stiffness and damping:

$$
F_{C}=k x+c \dot{x}, \quad x=u_{1}-u_{2}-d_{0} \quad \text { if } \quad k x+c \dot{x}>0 .
$$

Differentiation of the definition of the contact force over time yields:

$$
\frac{d F_{C}}{d t}-k \frac{d\left(u_{1}-u_{2}-d_{0}\right)}{d t}-c \frac{d^{2}\left(u_{1}-u_{2}\right)}{d t^{2}}=0
$$

At time instant when relative velocity of the colliding objects equals zero, the second term of Eq. (19) vanishes and the third term is positive (acceleration of the mass is negative and acceleration of the piston is positive). Therefore, the first term has to be negative and velocities of the objects equalize when contact force is decreasing, i.e. with certain delay to the peak of the contact force, Fig. 9(b). Similar situation occurs for the model of the contact force which involves nonlinear damping terms Eq. (3b).

\subsubsection{Real rubber contact material properties}

Rubber contact element used in the experiment is characterized by a complex mechanical behavior of rubber briefly described in Section 4.2. Precise modeling of rubber requires a three-dimensional model of the considered system as well as the Arruda-Boyce or the Ogden-Roxburg [29] constitutive models. The alternative approach proposed here does not require a numerical model and is based on experimental results only. The performed measurements indicate that the time instants at which the relative velocity vanishes can be determined with respect to the contact force peaks. The characteristic features of the process were observed:

- time shifts (i.e. $\left.\Delta t_{1}, \Delta t_{2}, \Delta t_{3}\right)$ were different for the first, second and third peaks of the contact force, cf. Figure 9(c),

- mentioned time shifts were almost independent of impacting mass and velocity.

The second of these features enables a straightforward application of the proposed method based on Eqs (14) and (15) since the duration of the time shifts are determined in advance. 


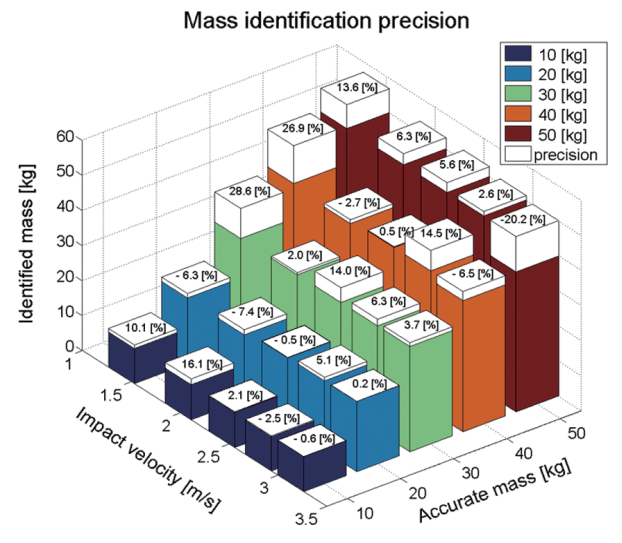

Fig. 10. Mass identification, experimental results (initial pressure $100 \mathrm{kPa}$ ).
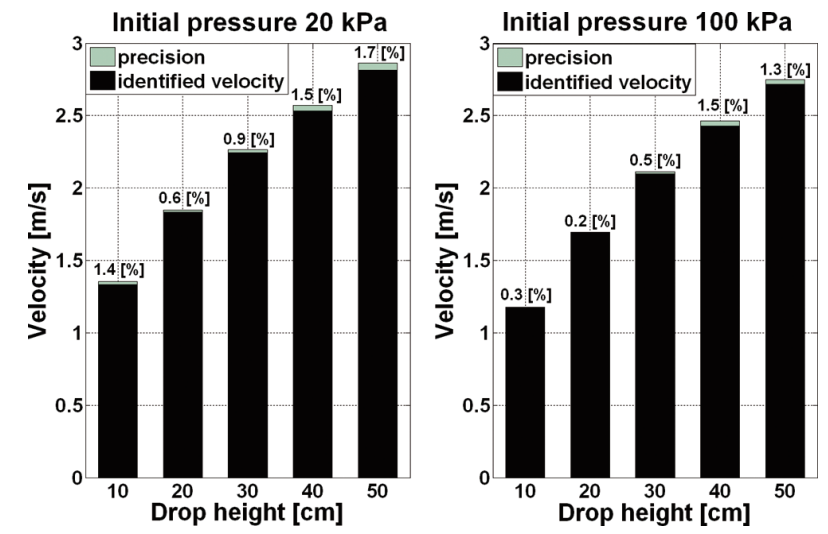

Fig. 11. Velocity identification, experimental results: (left) initial pressure 20 $\mathrm{kPa}$, (right) initial pressure $100 \mathrm{kPa}$.

\subsection{Verification of the method}

The proposed "peak to peak" identification method was verified experimentally and numerically. Equation (14) was applied in order to perform the mass identification. The experimental verification makes use of measurements from two sensors (i.e., force sensor and accelerometer attached to the piston rod). The initial experimental tests enable to obtain the average lengths of the time shifts $\Delta^{t 1}$ and $\Delta^{t 2}$ for the preselected set of impacts. They were further used during the stage of method verification for determination of the time instants $t^{01}$ and $t^{11}$.

The impact mass identification was tested for a vast variety of impact scenarios defined by impact mass, velocity and initial pressure. Exemplary results of identification in the case of the initial pressure of $100 \mathrm{kPa}$ are shown in Fig. 10. The graph presents in each case the exact value of the mass and the identification error (i.e. the relative difference between the identified and actual values).

The results presented in Fig. 10 show a large diversity of identification errors. It is a consequence of the strong sensitivity of the method to measurements inaccuracy which is especially apparent for small initial pressure and large mass of the impacting object.

The identification of the velocity was tested experimentally by using integration of the piston rod acceleration (see Eq. (15)). The method uses the assumption of the equality of velocities of the colliding objects at time instants $t^{01}$ and/or $t^{11}$. For verification purposes the instant $t^{11}$ was used, since then the impacting object mass is already identified. The determination of the actual velocity was performed by an analysis of a movie taken by the highspeed camera. The identification results are shown in Fig. 11. The accuracy is consistently better than $2 \%$ and no significant influence of the initial condition was observed.

The numerical model presented in Section 3 was used to perform a statistical analysis of mass identification error and to investigate the influence of selected parameters. The analyses were focused rather on qualitative than quantitative effects. In order to obtain more representative results, the average identification error for 125 impact cases (impact mass 10-50 kg, impact velocity 1-3 m/s, initial pressure $20-400 \mathrm{kPa}$ ) was calculated.

The proposed methodology turns out to be sensitive to impact conditions. Figure 12 presents the influence of impact mass, velocity and initial pressure on the mean identification error. More accurate results were obtained for smaller masses of the impacting object, which can be explained by an analysis of the ratio of the falling mass to the piston rod mass. A smaller ratio leads to a larger change of velocity of the dropped mass in the first phase of the impact process. As a consequence, the identification procedure seems to be less sensitive to measurements noise in the case of small impacting masses. The second aspect noticed from the statistical analysis is that a higher initial pressure in the pneumatic absorber leads to more accurate results. Contrary to the mentioned parameters, the velocity effect was not significant, even if the precision slightly increased together with the impact velocity.

The experimental results are often corrupted by the measurements noise. The sensitivity of the proposed methodology to these disturbances has been analyzed. In the numerical simulations, the noise-free simulation results were disturbed by random Gaussian noise in the range of $0-10 \%$, which was defined as the root mean square value of the 


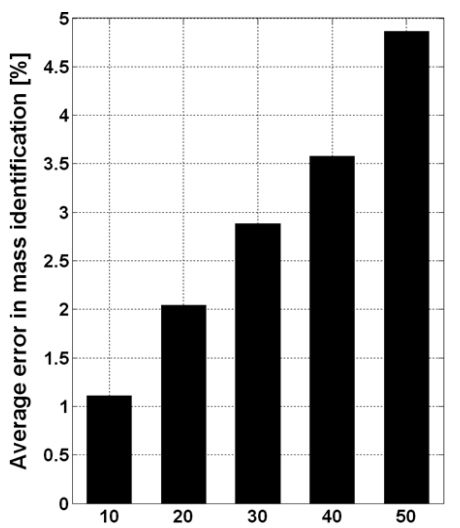

a.

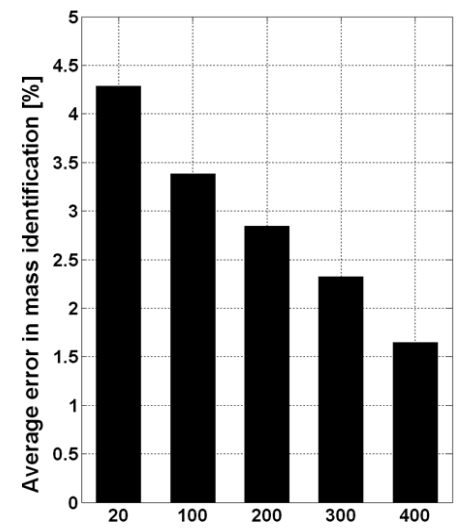

b.

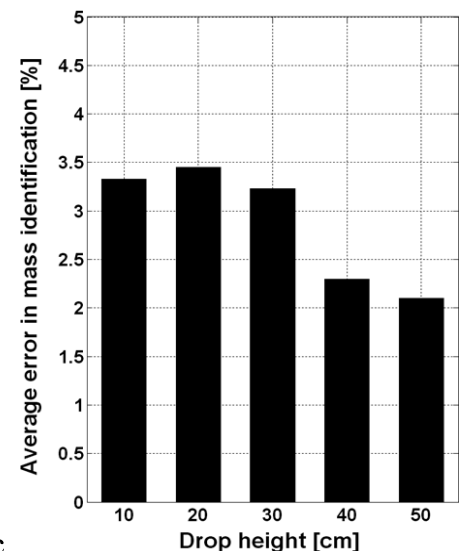

Fig. 12. Average error of mass identification in dependence on: a. falling mass; b. initial pressure; c. impact velocity.
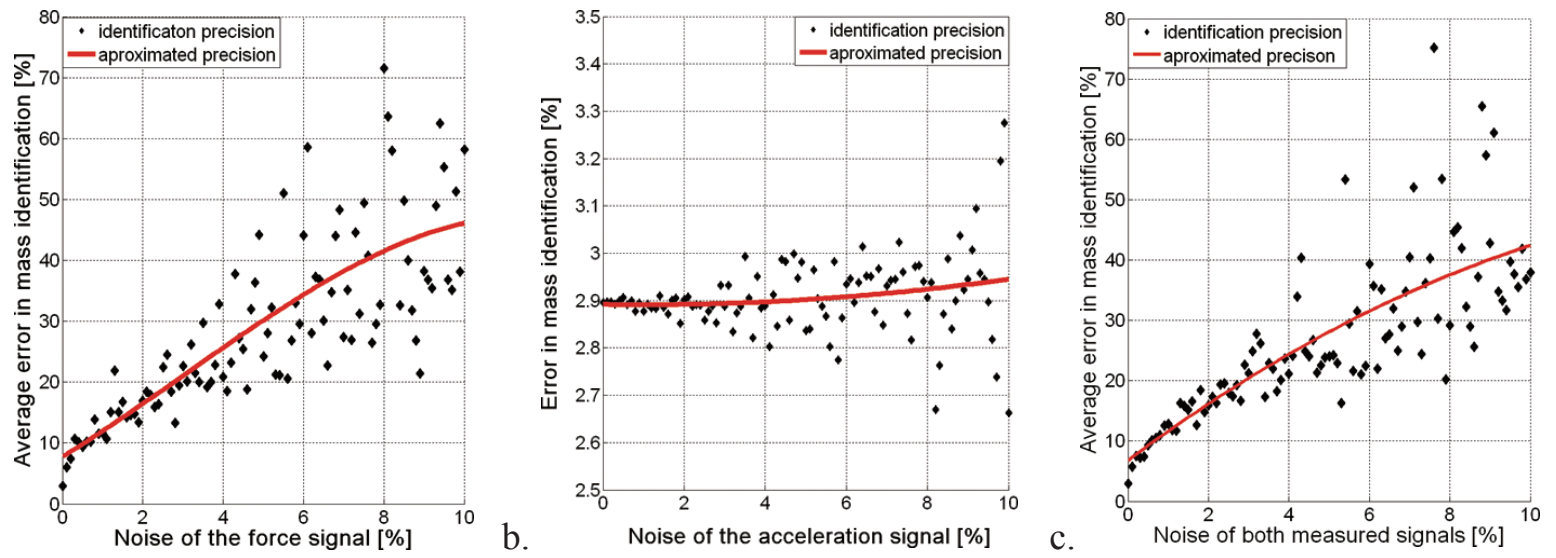

Fig. 13. Error of mass identification as a function of signal noise: a. force signal noise; b. acceleration signal noise; c. both signals noise.

original signal. The results are presented in Fig. 13, where the force, acceleration and both quantities were disturbed. Each point in the graph presents the mean identification error for 125 impact cases (defined by various masses, velocities and pressures). Despite the large number of tests, apparently random values of identification errors were obtained. The noise in the force signal was found to have a larger influence on mass identification error than the noise in the acceleration signal. It was noticed that the crucial task is the proper determination of the instants $t^{01}$ and $t^{11}$, which are obtained on the basis of the force signal. Hence, disturbances in the force signal lead to inaccurate determination of $t^{01}$ and $t^{11}$ and as a consequence, to errors in mass identification.

Finally, the influence of sampling frequency on the identification error has been analyzed. The results presented in Fig. 14 clearly reveal the importance of this parameter. As expected, higher sampling frequency enables to obtain more accurate results. Good results (i.e. mean error below 5\%) are obtained for the sampling frequencies exceeding 30-40 kHz. Unfortunately, even in this case extreme outliers (i.e. approx. 20\%) can be observed. A general conclusion might be drawn that the methodology requires a high sampling frequency of $50 \mathrm{kHz}$ or more.

\section{Response map approach}

The methodology proposed in this Section is focused on the maximum simplicity of the data acquisition set-up, i.e. application of an algorithm which utilizes measurements from one sensor only. Besides, the aim was to decrease the computational cost, which is crucial, as the device has to respond immediately in order to fulfill the deadline condition. 


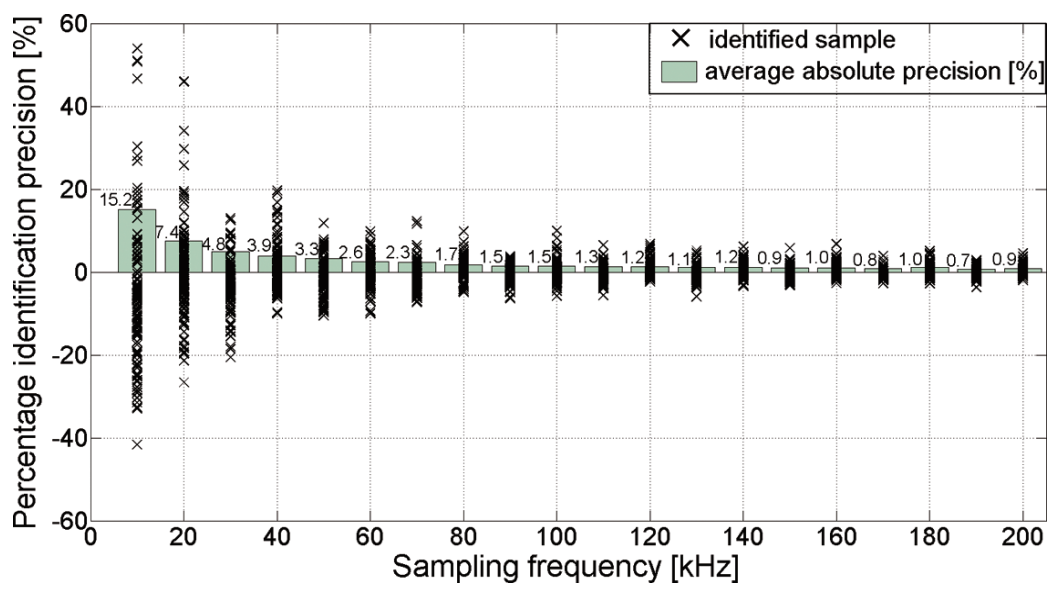

Fig. 14. Error of mass identification as a function of sampling frequency.
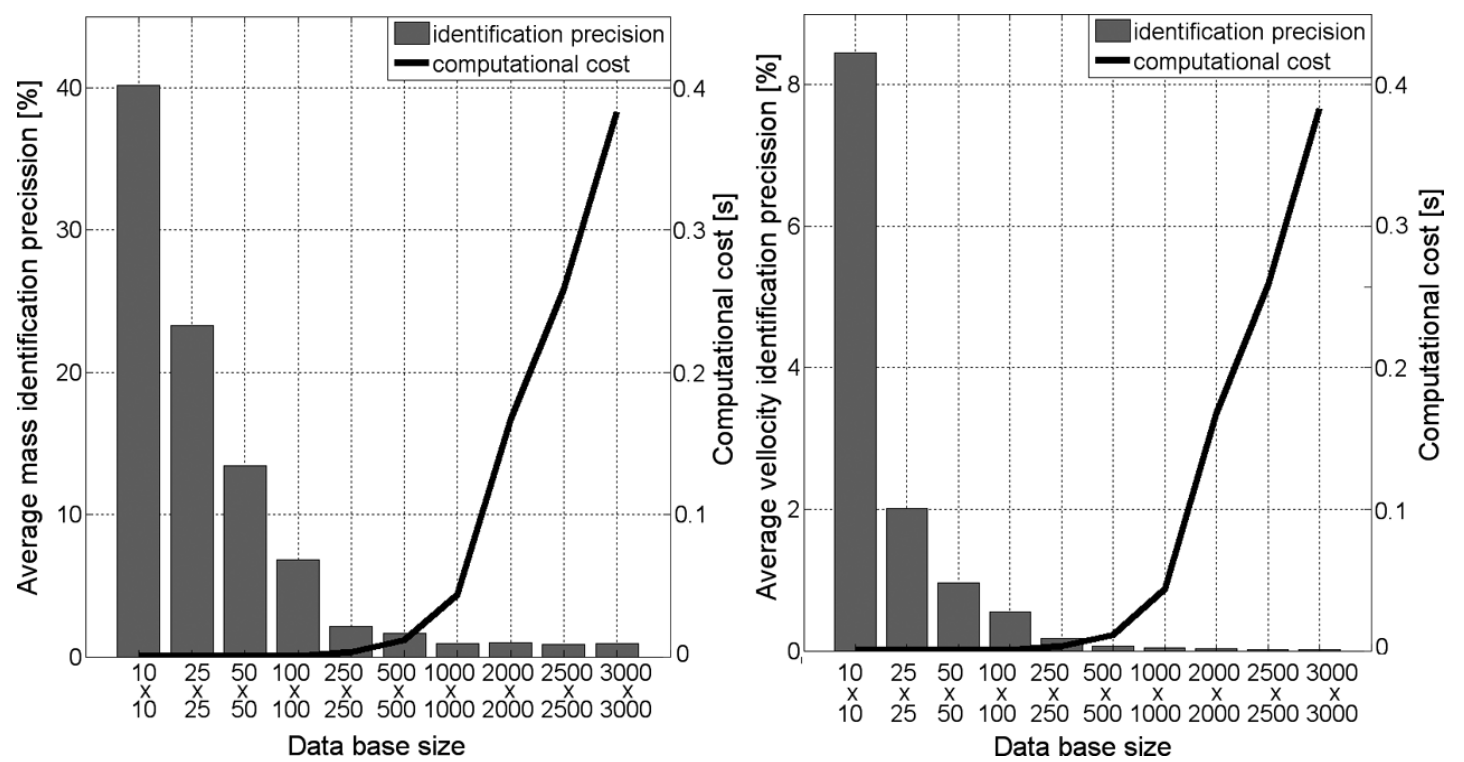

Fig. 15. Identification error and time as functions of database size: mass identification (left), velocity identification (right).

\subsection{The idea}

The proposed method can be classified into the group of pattern recognition techniques. The identification is performed based on a formerly prepared database of measured dynamic responses caused by various impact scenarios applied to the considered structure. The actually measured dynamic response is compared with the responses stored in the database. The methodology can be classified as model-free because the structural model is not required in the identification stage.

The objective of identification is to determine the impact parameters (mass and initial velocity of the impacting body) for which the structural response is the most similar to the actually measured response. The identification is based on a pre-fetched database (called the response map) which gathers selected characteristic parameters of the measured structural response $Y_{1}, Y_{2}, \ldots, Y_{m}$ (e.g. signal amplitude, its period etc.) that correspond to various parameters of the applied loading $x_{1}, x_{2}, \ldots, x_{n}$ (e.g. mass, impact velocity, etc.). General dependency of the structural response on impact parameters is defined by the following relations: 


$$
\begin{aligned}
& Y_{1}=Y_{1}\left(x_{1}, x_{2}, \ldots, x_{n}\right), \\
& Y_{2}=Y_{2}\left(x_{1}, x_{2}, \ldots, x_{n}\right), \\
& \ldots \ldots \\
& Y_{m}=Y_{m}\left(x_{1}, x_{2}, \ldots, x_{n}\right) .
\end{aligned}
$$

The approach consists of two main steps. In the first step, the database is generated, which can be performed either via a calibrated numerical model of the structure [30] or by experimental tests. The second step is the actual identification. Measurement of the actual response is performed and compared with the measurements stored in the database.

The proposed approach leads to an optimization problem in which the discrepancy between the actually measured response $Y_{i}^{M}$ and the stored responses $Y_{i}$ is minimized. With the normalized least squares discrepancy measure, the objective function to be minimized takes the following form:

$$
L\left(x_{1}, x_{2}, \ldots, x_{n}\right)=\sum_{i=1}^{m}\left[\frac{Y_{i}^{M}-Y_{i}\left(x_{1}, x_{2}, \ldots, x_{n}\right)}{Y_{i}^{M}}\right]^{2},
$$

and the corresponding optimization problem is to minimize of the objective function over impact parameters $x_{1}, x_{2}, \ldots, x_{n}$. The number of parameters to be identified, denoted by $n$, is limited by the number $m$ of the parameters used for identification: $n \leqslant m$.

In the considered case two parameters (i.e. falling objects mass $M_{1} \equiv x_{1}$ and its impact velocity $V_{1} \equiv x_{2}$ ) had to be identified based on a single measurement from the force sensor. Therefore, in order to construct the response maps, at least two characteristic quantities had to be extracted from each single measurement.

For the given response maps, the impact identification procedure utilizes the error function defined as:

$$
f\left\langle M_{1}, V_{1}\right\rangle=\left[\frac{Y_{1}^{M}-Y_{1}\left(M_{1}, V_{1}\right)}{Y_{1}^{M}}\right]^{2}+\left[\frac{Y_{2}^{M}-Y_{2}\left(M_{1}, V_{1}\right)}{Y_{2}^{M}}\right]^{2}
$$

where: $M_{1}$ and $V_{1}$ are the impact parameters to be identified, $Y_{1}\left(M_{1}, V_{1}\right)$ and $Y_{2}\left(M_{1}, V_{1}\right)$ are obtained from the response maps for the specific values of $M_{1}$ and $V_{1}$, while, $Y_{1}^{M}$ and $Y_{2}^{M}$ denote the actually measured values.

\subsection{Selection of parameters for the response maps}

An important task is the extraction of the characteristic features of the measured response to be stored in the database. Appropriate choice of these parameters (signal features) facilitates the identification and leads to more accurate results.

The response map is prepared based on the experimental measurements of the contact force. The choice of force sensor was motivated by its location (i.e. fixed to the absorber) and signal properties (smooth and not requiring filtering). However, the procedure can use other sensor, if its measurements is sensitive enough to the impact parameters.

Since the procedure utilizes contact force as a main quantity for the identification, its sensitivity on impacting mass and velocity is crucial for the effectiveness of the methodology. The analysis (see Section 4, Figs 3 and 6) conducted via the experimental measurements and numerical simulation proved strong dependence of the contact force on hitting object velocity during the whole period of impact. On the contrary, the dependence on mass of the impacting object is noticeable only in the second stage of impact. It indicates that in the first impact stage it is easier to identify the impacting object velocity than its mass.

As an example, the first parameter of the response $\left(Y_{1}\right)$ can be defined as amplitude of the first peak of the contact force, and the second parameter $\left(Y_{2}\right)$ as time integral of contact force in certain period of time $\left\langle 0, t_{\text {end }}\right\rangle$ :

$$
Y_{1}\left(M_{1}, V_{1}\right)=\max F_{C}\left(M_{1}, V_{1}, t\right), \quad t \in\left(t_{00}, t_{02}\right) ; \quad Y_{2}\left(M_{1}, V_{1}\right)=\int_{0}^{t_{\text {end }}} F_{C}\left(M_{1}, V_{1}, t\right) d t
$$



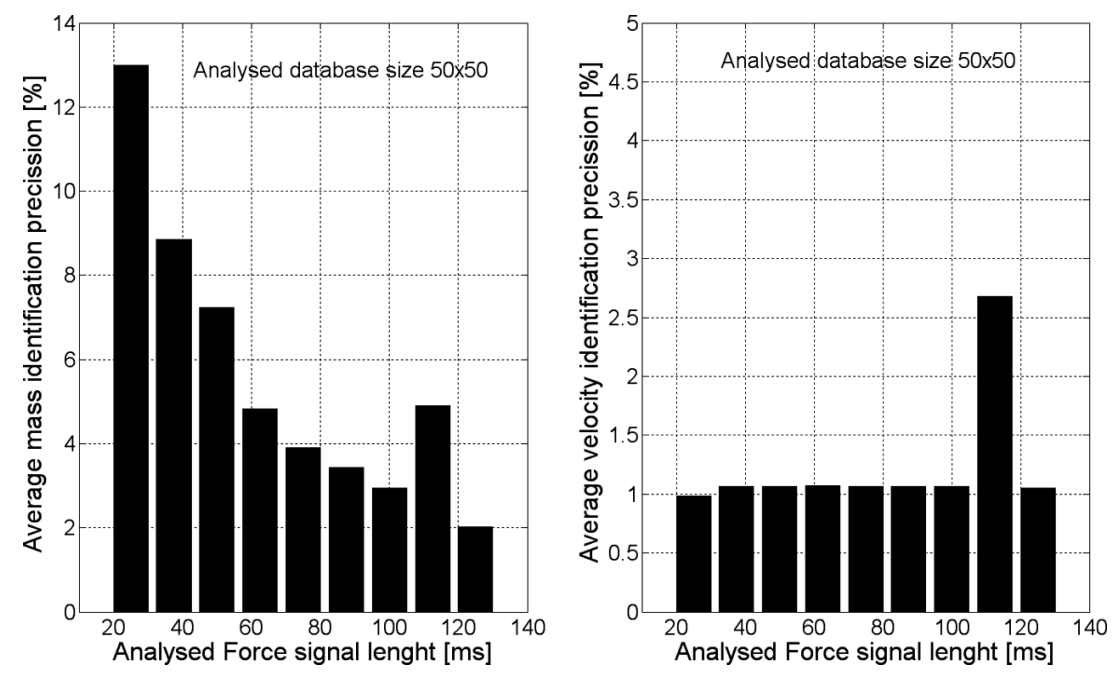

Fig. 16. Identification error as a function of analysed force signal length: mass identification (left), velocity identification (right).

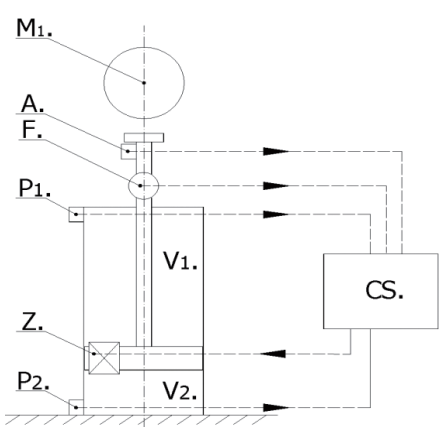

(a)

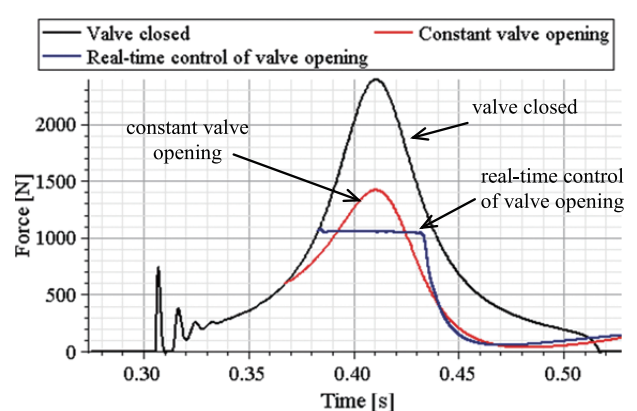

(b)

Fig. 17. An adaptive impact absorption system equipped with the Impactometer device a. schema of the proposed system b. numerical example of an impact scenario.

$$
Y_{1}^{M}=\max F_{C}^{M}(t), \quad t \in\left(t_{00}, t_{02}\right) ; \quad Y_{2}^{M}=\int_{0}^{t_{\text {end }}} F_{C}^{M}(t) d t
$$

The parameters defined by Eq. (23a) correspond to the values stored in the database while the parameters defined by Eq. (23b) are extracted from the actual measurement.

The choice of proper parameters of the response for preparation of the response map is also motivated by practical aspects. The selected values have to be characteristic for the impact process, i.e. it should be possible to unambiguously determine them from the measured signal (without neglecting the deadline condition). Hence, the objective is to find characteristic parameters of the response which exist at the beginning of the impact process (stage A). Moreover, the computational cost is significantly lower in case of a small-dimensional database. Sampling of the parameter space might be uniform or non-uniform. If a region of more frequent cases can be determined, the probability density functions can be used for sampling of the parameters space [30]. In the considered case it is assumed that each impact scenario is equally probable and a uniform sampling is applied.

Finally, taking into account all the above indications, the selected parameters were either the amplitudes of the two first peaks of the force signal or, alternatively, maximum value of force and its time integral in the considered time interval. 


\subsection{Verification of response map approach}

The verification of the method was focused on the sensitivity analysis and it was performed on the basis of numerical simulations. Two parameters were considered: identification error and computational cost.

The response map was built using the results obtained for 100 different impact scenarios (10 impacting masses $10-50 \mathrm{~kg}$ and 10 impact velocities determined by the drop height $5-50 \mathrm{~cm}$ ), while the initial pressure was not altered. Hence, the database was originally of the size $10 \times 10$ and utilized two parameters. The approach was tested using 10 randomly selected impacts. An important problem to be considered is the minimal size of the database which guarantees acceptable identification accuracy. The database density was numerically increased by interpolation. Nine databases $(25 \times 25,50 \times 50,100 \times 100,250 \times 250,500 \times 500,1000 \times 1000,2000 \times 2000,2500 \times 2500$, and $3000 \times 3000$ ) were obtained.

The graphs shown in Fig. 15 present the average identification error and the computational cost of identification of impacting mass Fig. 15(a) and impact velocity Fig. 15(b) as functions of the database size. Here, amplitudes of the first two peaks of the contact force in the first stage of the impact process were used as characteristic parameters. In each case, the accuracy of velocity identification turns out to be much higher (about 5-10 times) than the accuracy of mass identification. It is the result of the different sensitivity of the measured contact force on both parameters. In general, the accuracy of the response map approach increases together with the database size. Nevertheless, the computation time increases significantly as well. Hence, the identification process for large databases became impractical with regard to the deadline condition. For databases larger than $3000 \times 3000\left(9 \cdot 10^{6}\right.$ of mass-velocity variants) the computational cost exceeded $350 \mathrm{~ms}$ for a modern PC. As a consequence, the $500 \times 500$ database has been used to guarantee an acceptable identification accuracy and to keep the computational cost low.

Next, the maximum value of the contact force and its integral in a certain time interval were used as characteristic parameters to build the database. A significant influence of the integration interval length on the identification error was found. The results obtained for the database $50 \times 50$ are shown in Fig. 16. The accuracy increases together with the length of the time interval and this effect is the most significant for smaller databases. On a contrary, in the case of velocity identification, the obtained precision is insensitive to the length of the integration interval.

\section{Concept of an AIA device}

The conducted research enables to propose a concept of the device which utilizes the developed on-line identification methods. Such a device contains a gas spring, which can be switched to a pneumatic absorber (via controlled opening of the valve), and thus can be capable of impact energy absorption in a controllable manner. A simplified scheme of the impactometer together with the pneumatic AIA (adaptive impact absorption) device is shown in Fig. 17. The force sensor $F$ and the accelerometer $A$ are used for the identification of impact parameters and together with the gas spring and the control system CS, are the main part of the impactometer. The pressure sensors $P_{1}$ and $P_{2}$ are used for the measurement of the pressure in the chambers $V_{1}$ and $V_{2}$ and $V_{2}$ and together with the control system CS and controllable valve $\mathrm{Z}$ are the main parts of the AIA system.

The theoretical effectiveness of an AIA system which utilizes impactometer-based impact identification is shown in Fig. 17(b). Three force histories are presented: i) the case when the valve remains closed during the impact process, ii) the case with constant optimum valve opening and finally iii) the case with a real-time control of the gas flow. Both considered adaptation strategies enable to reduce the contact force observed in the second stage of collision and so to decrease the acceleration acting on the colliding object.

\section{Summary}

The paper presents a thorough analysis of the process of a rigid body impact into a pneumatic cylinder. The impact drop tests were performed experimentally and a numerical model of the impact process was developed. A wide variety of impact scenarios have been tested and its range has been numerically extrapolated. Various properties of contact element in collision region were investigated. Two algorithms for real-time impact load (i.e. impact mass, 
velocity) identification have been demonstrated (in Sections 5 and 6). Both algorithms operate in real-time and enable identification of impact during the initial milliseconds. It makes them useful for potential future applications in adaptive impact absorbing systems.

The first of the proposed approaches (the "peak-to-peak" method) uses two sensors (contact force and piston acceleration) for a very fast identification, which is possible due to the simplicity of the procedure. On the other hand, the method requires high sampling frequency and almost noise-free measurement data. Precision of the identification was found to be sensitive to the internal parameters of the impactometer. An acceptable precision was obtained when the mass of the impacting object was comparable to the mass of the piston rod (within the range of one order of magnitude) and for high pressures inside the cylinder.

The second of the proposed methods (the "response-map" approach) is based on single measurement only (contact force), however it requires initial preparation of the database. It can be obtained either by multiple experimental tests or by numerical simulations. The "response-map" approach requires longer identification time than the "peak-topeak" approach, but the results are more precise. The mean value of identification errors as well as their deviations decrease for larger databases, however at the cost of the identification time. It was proved that the precision is significantly improved by considering a longer contact force history, but it delays the identification process. Independently on the identification method, accurate velocity identification is much easier to perform than accurate mass identification.

In the next step, the impactometer will be realized and implemented. The problem of impact identification will be extended into two and three dimensional cases which is much more challenging and requires more advanced identification techniques.

\section{Acknowledgments}

This work was supported by the Polish research projects "Active control of the electro-mechanical drive systems of machines in unsteady operating conditions by means of actuators with the magneto-rheological fluid"ENERGOUDAR PBR - N R03 0012 and "Health Monitoring and Lifetime Assessment of Structures" - MONIT POIG.01.01.02-00-013/08-00.

Financial support of Structural Funds in the Operational Programme - Innovative Economy (IE OP) financed from the European Regional Development Fund - Project "Modern material technologies in aerospace industry", Nr POIG.01.01.02-00-015/08-00 is gratefully acknowledged. The presented paper is a part of the PhD theses by the first and the second author, which are supervised by the third author.

\section{References}

[1] J. Holnicki-Szulc, editor, Smart Technologies for Safety Engineering, Wiley, 2008, ISBN 978-0-470-05846-6(HB).

[2] G. Mikułowski, Adaptive impact absorbers based on magnetorheological fluids, PhD thesis, Institute of Fundamental Technological Research, 2008.

[3] X. Zhang, Conceptual study of adaptive energy absorbers, $\mathrm{PhD}$ thesis, The Hong Kong University of Science and Technology, 2009.

[4] J. Holnicki-Szulc and L. Knap, Adaptive crashworthiness concept, International Journal of Impact Engineering 30(6) (2004), 639-663, Doi: 10.1016/j.ijimpeng.2003.08.004.

[5] L. Knap, Aktywne sterowanie energii zderzeń w ustrojach adaptacyjnych, PhD thesis, Institute of Fundamental Technological Research, 2000.

[6] G. Mikułowski and Ł. Jankowski, Adaptive Landing Gear: optimum control strategy and potential for improvement, Shock and Vibration 16(2) (2009), 175-194, Doi: 10.3233/SAV-2009-0460.

[7] C. Graczykowski and J. Holnicki-Szulc, Protecting offshore wind turbines against ship impacts by means of adaptive inflatable structures, Shock and Vibration 16 (2009), 335-353, Doi: 10.3233/SAV-2009-0473.

[8] M. Ostrowski, P. Griskevicius and J. Holnicki, Pyro-adaptive impact energy absorber, Proc. of the Polish conference: Odporność udarowa konstrukcji, (2006) December 5-8, Rynia near Warsaw, Poland.

[9] M. Wiklo and J. Holnicki-Szulc, Optimal design of adaptive structures: Part I. remodeling for impact reception, Structural and Multidisciplinary Optimization 37 (2009), 305-318, Doi: 10.1007/s00158-008-0233-8.

[10] M. Wiklo and J. Holnicki-Szulc, Optimal design of adaptive structures: Part II. Adaptation to impact loads, Structural and Multidisciplinary Optimization 37 (2009), 351-366, Doi: 10.1007/s00158-008-0242-7.

[11] W.J. Witteman, Improved vehicle crashworthiness design by control of the energy absorption for different collision situations, PhD thesis, Eindhoven University of Technology, 1999, ISBN 90-386-0880-2. 
[12] J. Holnicki-Szulc, P. Pawlowski and M. Wiklo, High-performance impact absorbing materials-the concept, design tools and applications, Smart Materials and Structures 12 (2003), 461-467, Doi: 10.1088/0964-1726/12/3/317.

[13] S.S. Deshmukh and G.H. McKinley, Adaptive energy-absorbing materials using field-responsive fluid-impregnated cellular solids, Smart Materials and Structures 16 (2007), 106-113, Doi: 10.1088/0964-1726/16/1/013.

[14] G. Mikułowski and J. Holnicki-Szulc, Adaptive landing gear concept-feedback control validation, Smart Materials and Structures 16 (2007), 2146-2158, Doi: 10.1088/0964-1726/16/6/017.

[15] C. Graczykowski and J. Holnicki-Szulc, Inflatable structures with controlled release of pressure for adaptive impact absorption, Proceeding of $19^{\text {th }}$ International Conference on Adaptive Structures and Technologies, Ascona, 2008.

[16] J. Holnicki-Szulc, C. Graczykowski, G. Mikulowski, A. Mróz and P. Pawłowski, Smart technologies for adaptive impact absorption, Solid State Phenomena 154 (2009), 187-194, Doi: 10.4028/www.scientific.net/SSP.154.187.

[17] J. Holnicki-Szulc, C. Graczykowski, G. Mikułowski, A. Mróz, M. Ostrowski and R. Wiszowaty, 2011 Adaptive impact absorption for safety engineering, International Conference on Shock and Impact Loads on Structures, November 2011, Fukuoka, Japan.

[18] H. Inoue, J.J. Harrigan and S.R. Reid, Review of inverse analysis for indirect measurement of impact force, Appl Mech Rev 54 (2001), 503-524, Doi: 10.1115/1.1420194.

[19] M. Klinkov and C.P. Fritzen, An updated comparison of the force reconstruction methods, Key Engineering Materials 347 (2007), $461-$ 466, Doi: 10.4028/www.scientific.net/KEM.347.461.

[20] M.T. Martin and J.F. Doyle, Impact force identification from wave propagation responses, International Journal of Impact Engineering 18 (1996), 65-77, document Doi: 10.1016/0734-743X(95)00022-4.

[21] Ł. Jankowski, Off-line identification of dynamic loads, Structural and Multidisciplinary Optimization 37 (2009), 609-623, Doi: $10.1007 / \mathrm{s} 00158-008-0249-0$

[22] W.J. Stronge, Impact mechanics, Cambridge University Press, 2002; ISBN: 0-521-63286-2 (hb).

[23] N.W. Liepmann and A. Roshko, Elements of gasdynamics, John Wiley \& Sons New York 1957; ISBN: 0471534609.

[24] A.H. Shapiro, The dynamics and thermodynamics of compressible fluid flow, Pergamon Press, New York, 1953; ISBN: 082608060X.

[25] G.J. Van Wylen and R.E. Sonntag, Fundamentals of classical thermodynamics, John Wiley \& Sons, 1985; ISBN 10:0471800147.

[26] M. Sjöberg and L. Kari, Testing of nonlinear interaction effects of sinusoidal and noise excitation on rubber isolator stiffness, Polymer Testing 22 (2003), 343-351, document Doi: 10.1016/S0142-9418(02)00110-1.

[27] J. Diani, B. Fayolle and P. Gilormini, A review on the Mullins effect, European Polymer Journal 45 (2009): 601-612, document Doi: 10.1016/j.eurpolymj.2008.11.017.

[28] R. Gryboś, Teoria uderzenia w dyskretnych ukłdach mechanicznych, P. W. N., 1969.

[29] L.A. Gracia, E. Peńa, J.M. Royo, J.L. Pelegay and B.A. Calvo, Comparison between pseudo-elastic and damage models for modeling the Mullins effect in industrial rubber components, Mechanics Research Communications 36 (2009), 769-776.

[30] T. Szolc, P. Tauzowski, R. Stocki and J. Knabel, Damage identification in vibrating rotor-shaft systems by efficient sampling approach, Mech Syst Signal Process 47 (2009), 533-557, Doi: 10.1016/j.ymssp.2008.12.007.

[31] W.J. Staszewski, Intelligent signal processing for damage detection in composite materials, Composites Science and Technology 62 (2002), 941-950, document Doi:10.1016/S0266-3538(02)00008-8.

[32] D. Balageas, C.P. Fritzen and A. Güemes, Structural Health Monitoring, ISTE Ltd, 2006, ISBN 10: 1-905209-01-0.

[33] T. Uhl, The inverse identification problem and its technical application, Archive Applied Mechanics 77 (2007), 325-337, DOI: 10.1007/s00419-006-0086-9.

[34] J. LeClerc, K. Worden, W. Staszewski and J. Haywood, Impact detection in an aircraft composite panel- A neural-network approach, Journal of Sound and Vibration 299 (2007), 672-682, document Doi:10.1016/j.jsv.2006.07.019.

[35] G. Yan and L. Zhou, Impact load identification of composite structure using genetic algorithms, Journal of Sound and Vibration 319 (2009), 869-884, document Doi: 10.1016/j.jsv.2008.06.051

[36] H. Sekine and S. Atobe, Identification of locations and force histories of multiple point impacts on composite isogrid-stiffened panels, Composite Structures 89 (2009), 1-7, Doi: 10.1016/j.compstruct.2008.05.018. 

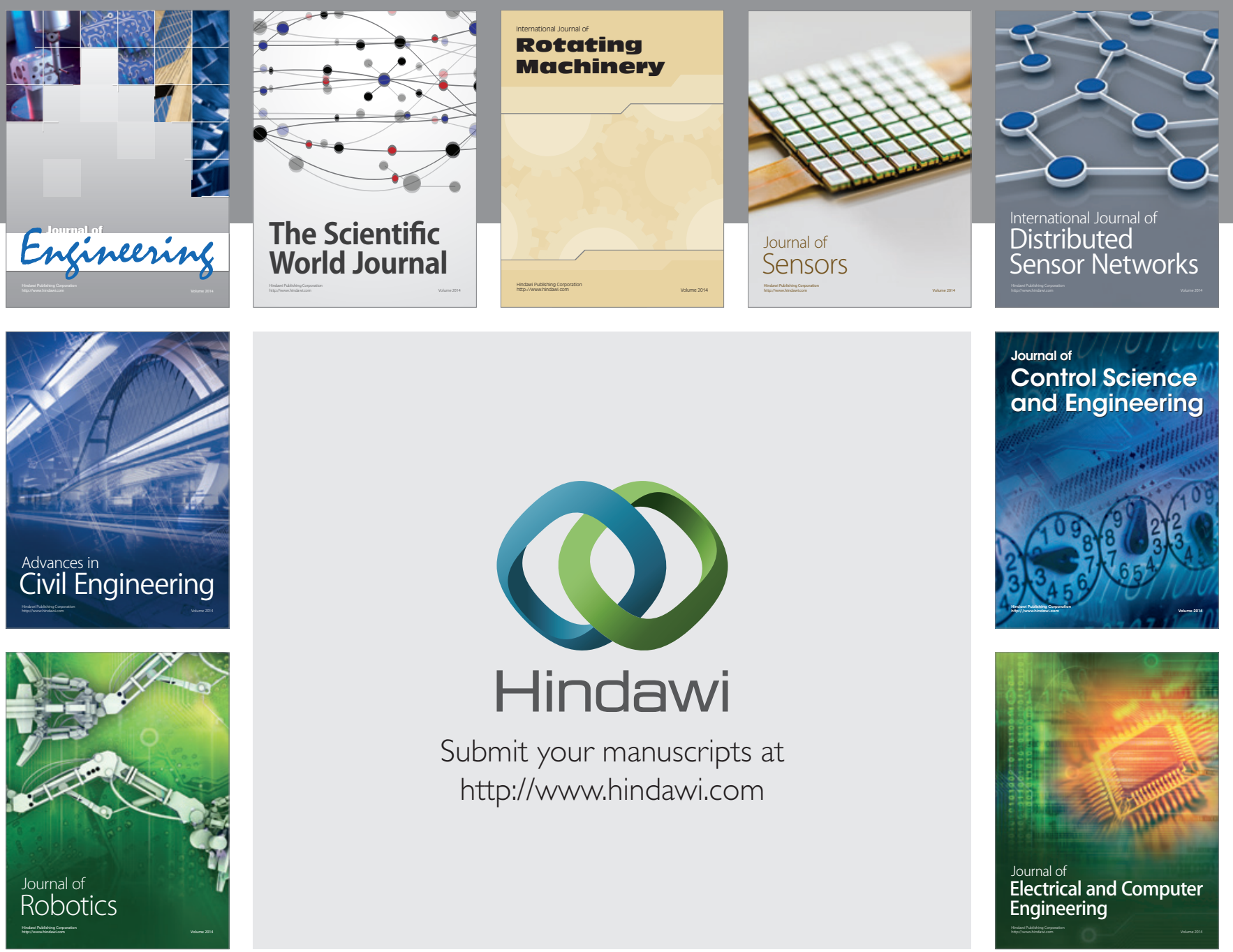

Submit your manuscripts at

http://www.hindawi.com
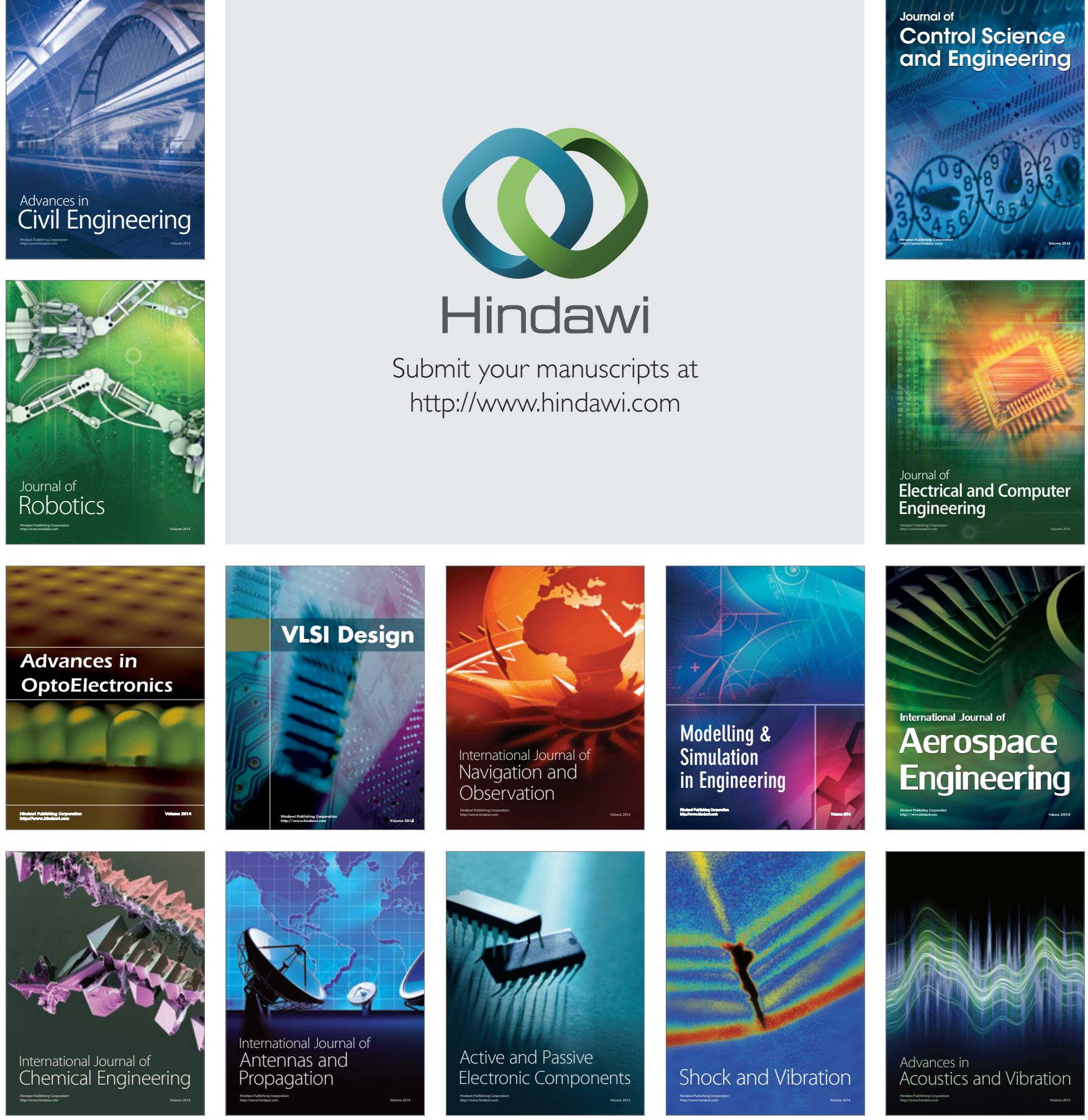